\title{
Urban Ecological Environmental Standards based on Subjective Evaluations of Various Environments
}

\author{
Lei YU \\ Shenzhen Graduate School of HIT \\ Shenzhen Key Laboratory of Urban planning and Decision \\ Making \\ Shenzhen, Guangdong, 518000, China \\ e-mail: leilayu@ hitsz.edu.cn
}

\author{
Hong LIANG \\ Shenzhen Environmental Monitoring Center \\ Center of Livable City's Ecology \& Environment \\ Shenzhen, Guangdong, 518000, China \\ e-mail: 376604225@qq.com
}

\author{
Dongchao XU \\ Shenzhen Graduate School of HIT \\ Shenzhen Key Laboratory of Urban planning and Decision \\ Making \\ Shenzhen, Guangdong, 518000, China \\ e-mail:626401805@qq.com
}

Jia'nan HOU

Shenzhen Graduate School of HIT

Shenzhen Key Laboratory of Urban planning and Decision Making

Shenzhen, Guangdong, 518000, China

e-mail: 87887729@qq.com

\begin{abstract}
With fast urbanization, urban nature environment has been largely damaged. A primary ecological system is being destroyed. In order to a sustainable development, environmental standards for maintain an ecological system for a city have to be established. This study demonstrates a new approach for establishing urban ecological environmental standards according to subjective environmental evaluations. Firstly, urban ecological environmental system and standards based on subjective evaluations have been established. Following that, field studies in some typical sites of the Shenzhen six administration districts have been made. Based on relationship analyses of various physical parameters and socioeconomic indices with subjective evaluations of various environment, it is found that each environment has its different influence physical parameters and socioeconomic indices but the nature of industrial land use is one of all of them. Except sound pressure level, no statistical significance has been found to physical parameters and socioeconomic indices on any environmental evaluation, in which further study with more samples is needed. Eventually, The results will be used to guide urban designers and planners theoretically in their practices.
\end{abstract}

Keywords-urban ecological environmental standards; subjective evaluations; various environments

\section{INTRODUCTION}

\section{A. Study Background}

With Chinese deepening urbanization and industrialization, more and more peoples from villages rush into cities. This immigration forces a city to make a vast constructions that have done a big damage on the original nature environment, which destroy its ecological system and makes the city's development no longer sustainable. Nowadays, it is realized that establishing proper urban ecological environmental standards is important to change the current developing situation and to improve the environmental qualities and to maintain ecological system [1-4]. Therefore, many models such as PSR (Pressure-StateRespond), DPSIR (Drive-Pressure-State-Impact-Respond), PSIR (Pressure-State-Impact-Respond), DSR (Drive-StateRespond) etc. are proposed and developed [5-8]. Although social and economic factors have been considered, the previous models are not taken human's perception into account, which is an essential standard to judge an ecological environment [9-12]. Therefore, this paper illustrates a study of using subjective evaluations to evaluate an ecological environment. As having a vast change from villages into a metropolis, Shenzhen has been used as an example to study urban ecological environmental standards according to subjective evaluations. In the study, subjective evaluations of various environments including air, water, noise and soil are explored based on a series field studies. Relationships of subjective evaluations of various environments and the physical parameters and socioeconomic indices are thoroughly explored. The results are supposed to be used as guidelines to urban design and planning for the city's sustainable development.

\section{B. Field Study Sites}

According to morphology and topology of the Shenzhen six administration districts, a series field study sites have been selected to study subjective evaluations of air, water, and noise environment with their physical index. The six administration districts are Bao An, Nan Shan, Fu Tian, Luo $\mathrm{Hu}$, Long Gang, and Yan Tian. Referring to urbanization, Luo Hu, Fu Tian, and Nan Shan are well developed, in which the Lou Hu contains more commercial service industries, Fu Tian functions more official industries, and Nan Shan has more high-end manufactures along with logistics industries although mixed industries exist to all of them. However, Bao An and Long Gang are not well urbanized districts that are in the fast developing stage. In these two districts, low-end 
manufactures are common. Compared to the other districts, Yan Tian is less developed mainly having logistics industries because of having a big seaport, therefore it is assumed having a better ecological environment than others. According to each district's characteristics, sixteen study sites are selected to launch a field study. Two study sites are selected in the Luo Hu, Bao An and Yan Tian respectively. Three study sites are selected in the Fu Tian and Long Gang district respectively. And four study sites are selected in the Nan Shan.

\section{RESEARCH METHOD}

In the study, six administration districts of Shenzhen, namely Yan Tian, Luo Hu, Long Gang, Fu Tian, Nan Shan and Bao An, have been firstly explored to obtain typical study sites for studying subjective evaluations of air, water, noise, and soil environment. Based on analyzing morphological characteristics of air, water, noise, and soil environment in each administration district, nine sites have been chosen to study evaluations of air and noise environment respectively, 8 sites have been chosen to study evaluations of water environment, and 4 sites have been chosen to study evaluations of soil environment. Totally, 16 study sites have been chosen, in which 2 in the Yan Tian, Luo $\mathrm{Hu}$ and Bao An respectively, 3 in the Fu Tian and Long Gang respectively, and 4 in the Nanshan. In order to elicit subjective evaluation of various environment, social surveys were carried out in these study sites and a questionnaire has been designed. It contains subjects' social and demographic information such as age, gender, occupation etc., their feelings of the study sites' air, water, noise, and soil environment. Besides, questions of personality preference on various environments' quality requirements are also asked. A 5 -scale measurement is used to define subjective evaluations of various environment, in which 1 stands for the worst and 5 stands for the best. In total, thirty interviews have been made to each study site and four hundred eighty answering sheets have been obtained.

The field studies were carried out in three continue days in order to be possible to make a comparison. Sixteen study sites in the Yan Tian, Long Gang, Luo Hu, Fu Tian, Nan Shan and Bao An have been explored. Social surveys were conducted in the study sites and the noise level was measured simultaneously. Physical parameters to the air and water environment of the studied sites are obtained from the nearby environmental monitoring stations of the Shenzhen environmental monitoring center. However, physical parameters of the soil environment cannot be got due to limitation policy. A reason of using global data instead of situ measurements is because the global data can express a variation of the environment, which is more precise to reflect the environments' influence on the individuals. To the socioeconomic indices, a $5 \mathrm{~km}^{2}$ range of the study site were calculated based on the publication documents of the Shenzhen government, which include economic development data (such as industrial type, Gross Domestic Product-GDP), land use data (nature of land use). The data of physical parameters were collected in the nearby monitoring stations less than $5 \mathrm{~km}^{2}$ away or situ measurements.

\section{RESULTS}

Subjective evaluation of various environment as important evaluation standard of urban ecological environment, their relationships of physical parameters and social indices are crucial in representing a city's ecological environmental circumstance. Through a case study of analyzing relationships of subjective environmental evaluations with physical parameters of the air, water, and noise environment and socioeconomic indices of the sixteen study sites in Shenzhen, this study is going to discover how urban natural and social factors influence subjective evaluations of an ecological environment and then to be able to establish urban ecological environmental standard in respect of peoples' feelings. By using SPSS [13], the relationships of the air, water and noise environmental evaluation with their physical reflecting parameters and related socioeconomic indices were systematically analyzed in the following sections.

\section{A. Air Environment}

In this section, subjective evaluations of air environment with its physical parameters including $\mathrm{SO}_{2}, \mathrm{NO}_{2}, \mathrm{NO}, \mathrm{PM} 10$, and PM2.5 were analyzed, as well as the socioeconomic indices, such as industrial type and nature of land use. The results are shown in the Table I. In the Table I, it can be seen that the subjective evaluations of air environment are not statistically significantly correlated with any physical parameters or socioeconomic indices although some rather high relationships exist. It is found that a high relation of $\mathrm{PM} 10, \mathrm{SO}_{2}$ and $\mathrm{NO}_{2}$ with the air environmental evaluations exists. A rather high relation of industrial type and nature of land use indices with the air environmental evaluations also exists. This means that these physical parameters and socioeconomic indices might influence the subjective evaluations of air environment. A reason of no significant correlation exists might be the subjects were not enough to do a statistic correlation analysis since a large number of samples are usually needed when doing subjective evaluation research. In the Table I, it can also be seen that some physical parameters are highly related with some socioeconomic indices even some reach a statistic significant, which implies that urban development would affect the physical characteristics of air environment. 


\begin{tabular}{|c|c|c|c|c|c|c|c|c|c|c|c|c|}
\hline & $\mathrm{AE}$ & PM10 & PM2.5 & $\mathrm{SO}_{2}$ & $\mathrm{NO}_{2}$ & NO & SI & TI & $\mathrm{IL}$ & TL & PL & GDP \\
\hline$\overline{\mathrm{AE}}$ & - & .32 & .07 & .62 & -.37 & .09 & .11 & -.11 & .25 & .11 & -.20 & .03 \\
\hline PM10 & .32 & - & $.89^{* *}$ & .65 & .63 & .08 & .65 & -.65 & $.88^{* *}$ & .59 & -.64 & .50 \\
\hline PM2.5 & .07 & $.89 * *$ & - & .30 & $.79^{*}$ & .38 & .54 & -.54 & .70 & .65 & $-.73^{*}$ & .52 \\
\hline $\mathrm{SO}_{2}$ & .62 & .65 & .30 & - & -.02 & -.07 & .57 & -.57 & .64 & -.00 & -.08 & .02 \\
\hline $\mathrm{NO}_{2}$ & -.37 & .63 & $.79 *$ & -.02 & - & .09 & .16 & -.16 & .38 & $.75^{*}$ & $-.78^{*}$ & .58 \\
\hline NO & .09 & .08 & .38 & -.07 & .09 & - & .25 & -.25 & -.07 & .06 & -.18 & -.03 \\
\hline SI & .11 & .65 & .54 & .57 & .16 & .25 & - & $-1.0^{* * *}$ & $.81^{* *}$ & .31 & -.30 & .56 \\
\hline $\mathrm{TI}$ & -.11 & -.65 & -.54 & -.57 & -.16 & -.25 & $-1.0^{* *}$ & - & $-.81^{* *}$ & -.30 & .30 & -.56 \\
\hline NIL & .25 & $.88^{* *}$ & .70 & .64 & .38 & -.07 & $.81^{* *}$ & $-.81^{* *}$ & - & .48 & -.48 & .61 \\
\hline TL & .11 & .59 & .65 & -.00 & $.75^{*}$ & .06 & .31 & -.31 & .48 & - & $-.98^{* *}$ & $.90^{* *}$ \\
\hline PL & -.20 & -.64 & $-.73^{*}$ & -.08 & $-.78^{*}$ & -.18 & -.30 & .30 & -.48 & $-.98^{* *}$ & - & $-.84^{* *}$ \\
\hline GDP & .03 & .50 & .52 & .02 & .58 & -.02 & .56 & -.56 & .61 & $.90^{* *}$ & $-.84^{* *}$ & - \\
\hline
\end{tabular}

AE-Air Environmental Evaluation, SI-Occupation Percentage of the Second Industry in the Whole Industry, TI-Occupation Percentage of the Tertiary Industry in the Whole Industry, NIL-Nature of Industrial Land Use, TL-Traffic Land Use, PL-Public Land Use, GDP-Gross Domestic Product

TABLE II. THE CORRELATION BETWEEN WATER COMPONENT, ECOLOGICAL AND SOCIO- ECONOMIC INDICATORS

\begin{tabular}{lccccccccccccc}
\hline & WE & T & CD & BOD & AN & M & C & SI & TI & IL & TL & PL & GDP \\
\hline WE & - & .31 & -.24 & -.60 & -.06 & -.27 & -.63 & -.02 & .02 & .17 & .03 & -.24 & -.17 \\
T & .31 & - & $-1.0^{*}$ & .00 & a. & a. & a. & -.63 & .63 & -.89 & $-1.0^{*}$ & 1.00 & -.95 \\
CD & -.24 & $-1.0^{*}$ & - & .00 & a. & a. & a. & .57 & -.57 & .85 & .99 & -.99 & .92 \\
BOD & -.60 & .00 & .00 & - & $.75^{*}$ & .56 & $.72^{*}$ & .09 & -.09 & -.10 & .42 & -.30 & .32 \\
AN & -.06 & a. & a. & $.75^{*}$ & - & .23 & .57 & .39 & -.39 & .38 & .46 & -.42 & .31 \\
M & -.27 & a. & a. & .56 & .23 & - & .03 & -.37 & .37 & -.34 & .37 & -.27 & .15 \\
C & -.63 & a. & a. & $.72^{*}$ & .57 & .03 & - & .23 & -.23 & -.05 & -.05 & .14 & -.05 \\
SI & -.02 & -.63 & .57 & .09 & .39 & -.37 & .23 & - & $-1.0^{* *}$ & $.89^{* *}$ & .16 & -.24 & .52 \\
TI & .02 & .63 & -.57 & -.09 & -.39 & .37 & -.23 & $-1.0^{* *}$ & - & $-.89^{* *}$ & -.16 & .24 & -.52 \\
NIL & .17 & -.89 & .85 & -.10 & .38 & -.34 & -.05 & $.89^{* *}$ & $-.89^{* *}$ & - & .28 & -.34 & .54 \\
TL & .03 & $-1.0^{*}$ & .99 & .42 & .46 & .37 & -.05 & .16 & -.16 & .28 & - & $-.96^{* *}$ & $.83^{* *}$ \\
PL & -.24 & 1.0 & -.99 & -.30 & -.42 & -.27 & .14 & -.24 & .24 & -.34 & $-.96^{* *}$ & - & $-.79^{* *}$ \\
GDP & -.17 & -.95 & .92 & .32 & .31 & .15 & -.05 & .52 & -.52 & .54 & $.83^{* *}$ & $-.79^{* *}$ & - \\
\hline
\end{tabular}

WE-Water Environmental Evaluation, T-Transparency of water body, CD-Cyanobacteria density, BOD-Biology Oxygen Demand, AN-Ammonia Nitrogen, M- Occupation of Mercury in the water body, C-Occupation of Cyanide in the water body, SI-Occupation Percentage of the Tertiary Industry in the Whole Industry, TI- Occupation Percentage of the Tertiary Industry in the Whole

\section{B. Water Environment}

In the section 3.2, subjective evaluations of water environment with the physical parameters of water transparency, cyanobacteria density, BOD (Biology Oxygen Demand), AN (Ammonia Nitrogen), mercury, and cyanide were analyzed, and also the same socioeconomic indices as analyzed in the air environment. The results are shown in the Table II. It is also found that no statistically significant correlation of physical parameters or socioeconomic indices with subjective evaluations of the water environment although some correlation is still high. It is found that a high relation of water transparency, cyanobacteria density, BOD, mercury, and cyanide with the water environmental evaluations exists. A high relation of some industrial type and nature of industrial land use indices and GDP (Gross Domestic Product) with the water environmental evaluations exists yet but less higher compared to the relations of the physical parameters. This might be the same reason of no significant correlation exists between any physical parameter or socioeconomic index with the water environmental evaluations as with the air environmental evaluations, which is the study does not supply enough samples to do a statistic correlation. Compared to the air environmental evaluation, more physical parameters but less socioeconomic indices have a high relation with the water environmental evaluation. In the Table II, it can also be seen that some physical parameters are highly related with some socioeconomic indices even some reach a statistic significant. It implies that urban development would affect the physical characteristics of water environment.

\section{Noise Environment}

In this section, relationships of noise environmental evaluations with sound pressure level and also the socioeconomic indices analyzed in the air and water environment were explored as shown in the Table III. It can be seen that except sound pressure level, no statistically significant correlation of socioeconomic indices with noise environmental evaluations although some high relationships exist. To the socioeconomic indices, a rather high relation of nature of land use indices with the noise environmental evaluations although not reaching a statistical significance. A same reason might exist of this as others in the air and water environmental evaluation studies due to no enough samples for analyses. An obvious difference of the noise 
environmental evaluations with the air and water environmental evaluations is the physical parameter of sound pressure level has a statistic significant influence on the subjective evaluations. In the Table III, it can also be seen that sound pressure level is highly related with some socioeconomic indices but not reaching a statistical significance. The result might be explained that urban development might influence the sound level of noise environment.

TABLE III. THE CORRELATION BETWEEN NOISE INDICATORS, ECOLOGICAL AND SOCIOECONOMIC INDICATORS

\begin{tabular}{lcccccccc}
\hline & QE & SL & SI & TI & IL & TL & PL & GDP \\
\hline QE & - & $-.64^{*}$ & .34 & -.34 & .37 & -.12 & .00 & .10 \\
SL & $-.64^{*}$ & - & -.09 & .09 & .00 & .25 & -.30 & -.09 \\
SI & .34 & -.09 & - & $-1.0^{* *}$ & $.85^{* *}$ & .21 & -.25 & .50 \\
TI & -.34 & .09 & $-1.0^{* *}$ & - & $-.85^{* *}$ & -.21 & .25 & -.50 \\
NIL & .37 & .00 & $.85^{* *}$ & $-.85^{* *}$ & - & .39 & -.43 & .57 \\
TL & -.12 & .25 & .21 & -.21 & .39 & - & $-.97^{* *}$ & $.89^{* *}$ \\
PL & .00 & -.30 & -.25 & .25 & -.43 & $-.97^{* *}$ & - & $-.85^{* *}$ \\
GDP & .10 & -.09 & .50 & -.50 & .57 & $.89^{* *}$ & $-.85^{* *}$ & - \\
\hline \multicolumn{7}{c}{ QE-Quiet Evaluation, SL-Sound Pressure Level, SI-Occupation Percentage of the } \\
Tertiary Industry in the Whole Industry, TI-Occupation Percentage of the Tertiary \\
Industry in the Whole Industry, IL-Nature of Industrial Land Use, TL-Traffic Land, PL- \\
\multicolumn{8}{c}{ Public Land, GDP-Gross Domestic Product }
\end{tabular}

\section{CONCLUSION}

Based on a case study of Shenzhen, this study found that except sound pressure level to the noise environmental evaluation, all other physical parameters and socioeconomic indices have no statistic significant influence on the subjective evaluations although some high relationship exists. A possible reason of this is that not enough study samples used in the study to analyze subjective evaluations due to its complication. In general, it is found that PM10, $\mathrm{SO}_{2}$ and $\mathrm{NO}_{2}$ are highly related with the air environmental evaluation, while water transparency, cyanobacteria density, BOD and mercury are highly related with the water environmental evaluation, and the sound pressure level is highly related with the noise environmental evaluation and reaches a statistical significance. In the meanwhile, many socioeconomic indices of industrial type and nature of land use are also highly related with the air, water, and noise environmental evaluations; however, compared to the physical parameters, their relationships are less. To the socioeconomic indices, it is interesting to find that nature of industrial land use is highly related with all the three environmental evaluations indicating it might be an important socioeconomic index to urban ecological environmental standard.

\section{REFERENCES}

[1] Dalsgaard J P T. An ecological modeling approach towards the determination of sustainability in farming system [J]. Royal Agriculture and Veterinary, (1996).

[2] Kane C T, Wolfe M A, Dale A K, Suter V H, Russell G W, Pion C S, Hadley G M, Konar V C. Communicating ecological indicators to decision-makers and the public [J]. Conservation Eole, 5(1):19 (2001).

[3] Dalsgaard J P T, Lightfoot C, Christensen V. Towards quantification of ecological sustainability in farming systems analysis [J]. Ecological Engineering, (1995).

[4] Virginia H, Suzanne D, Beyeler C. Challenges in the development and use of ecological indicators [J]. Ecological indicators, (2001).

[5] Tong Chuan. Review on environmental indicator research [J]. Research On Environmental Science, 13(4):53 (2000). (In Chinese)

[6] European Environment Agency. Europe's Environment: The Second Assessment [R]. Oxford: Elsevier Science Ltd, (1998).

[7] Yingxin Tan, Zhongxiang Yu. An Ecological Security Evaluation of Huaihe River Based on DSR [J]. Journal of Anhui Agricultural University, (2012 ). (In Chinese)

[8] Zongjun Wang, Wenyan Pan. Comprehensive Evaluation on Low Carbon Economy: Based on Driver-Pressure-State-Impact-Response Model. Technology Economics, (2012). (In Chinese)

[9] Zongqing Zheng. Urban ecotope quality evaluation in Guangzhou. Journal of Shanxi Normal University, (1995). (In Chinese)

[10] Wanxin Wang, Zhaoli Jiang, Yi Liu. An research into the factor pattern and methods for the appraisal of chengdu urban ecological environment quality. Journal of Sichuan Normal University, (1996). (In Chinese)

[11] Bentai Wan, Wenjie Wang, Shuhong Cui,Yingzi Pan, Jianhui Zhang. Research on the methods of urban ecological environmental quality assessment [J]. Acta Ecologica Sinica, (2009). (In Chinese)

[12] Jing Song, Huixiao Wang, Fei Wang. Research progress of ecological environment quality assessment and methods review $[\mathrm{J}]$. Environment Science \& Technology, (s2): 448-453 (2013). (In Chinese)

[13] Dunn P. SPSS survival manual: a step by step guide to data analysis using IBM SPSS [J]. Passenger Transport, 37(6): 597-598 (2010). 